12. Condorelli, G., et al. 2002. Akt induces enhanced myocardial contractility and cell size in vivo in transgenic mice. Proc. Natl. Acad. Sci. U. S. A. 99:12333-12338.

13. Shioi, T., et al. 2002. Akt/protein kinase B promotes organ growth in transgenic mice. Mol. Cell. Biol. 22:2799-2809.

14. Nagoshi, T., et al. 2005. PI3K rescues the detrimental effects of chronic Akt activation in the heart during ischemia/reperfusion injury. J. Clin. Invest. 115:2128-2138. doi:10.1172/JCI23073.

15. Shiojima, I., et al. 2005. Disruption of coordinated cardiac hypertrophy and angiogenesis contributes to the transition to heart failure. J. Clin. Invest. 115:2108-2118. doi:10.1172/JCI24682.

16. McMullen, J.R., et al. 2003. Phosphoinositide 3-kinase(p110alpha) plays a critical role for the induction of physiological, but not pathological, cardiac hypertrophy. Proc. Natl. Acad. Sci. U. S. A. 100:12355-12360.
17. McMullen, J.R., et al. 2003. The insulin-like growth factor 1 receptor induces physiological heart growth via the phosphoinositide 3-kinase(p110alpha) pathway. J. Biol. Chem. 279:4782-4793.

18. Ackah, E., et al. 2005. Akt1/protein kinase $B \alpha$ is critical for ischemic and VEGF-mediated angiogenesis. J. Clin. Invest. 115:2119-2127. doi:10.1172/ JCI24726.

19. Shioi, T., et al. 2000. The conserved phosphoinositide 3-kinase pathway determines heart size in mice. EMBO J. 19:2537-2548.

20. Yamashita, K., et al. 2001. Reperfusion-activated Akt kinase prevents apoptosis in transgenic mouse hearts overexpressing insulin-like growth factor-1. Circ. Res. 88:609-614

21. McMullen, J.R., et al. 2004. Deletion of ribosomal S6 kinases does not attenuate pathological, physiological, or insulin-like growth factor 1 receptorphosphoinositide 3-kinase-induced cardiac hypertrophy. Mol. Cell. Biol. 24:6231-6240.
22. McMullen, J.R., et al. 2004. Inhibition of mTOR signaling with rapamycin regresses established cardiac hypertrophy induced by pressure overload. Circulation. 109:3050-3055.

23. $\mathrm{Hu}, \mathrm{P}$., et al. 2003. Minimally invasive aortic banding in mice: effects of altered cardiomyocyte insulin signaling during pressure overload. Am. J. Physiol. Heart Circ. Physiol. 285:H1261-H1269.

24. Mora, A., et al. 2003. Deficiency of PDK1 in cardiac muscle results in heart failure and increased sensitivity to hypoxia. EMBO J. 22:4666-4676.

25. White, M.F. 2002. IRS proteins and the common path to diabetes. Am. J. Physiol. Endocrinol. Metab. 283:E413-E422.

26. Kim, Y.K., et al. 2003. Mechanism of enhanced cardiac function in mice with hypertrophy induced by overexpressed Akt. J. Biol. Chem. 278:47622-47628.

27. Morisco, C., et al. 2005. Akt mediates the cross-talk between beta-adrenergic and insulin receptors in neonatal cardiomyocytes. Circ. Res. 96:180-188.

\title{
A new direction for gene therapy: intrathymic T cell-specific lentiviral gene transfer
}

\author{
Ruth Seggewiss and Cynthia E. Dunbar
}

National Heart, Lung, and Blood Institute, NIH, Bethesda, Maryland, USA.

\begin{abstract}
Reports of neoplasia related to insertional activation of protooncogenes by retroviral vectors have raised serious safety concerns in the field of gene therapy. Modification of current approaches is urgently required to minimize the deleterious consequences of insertional mutagenesis. In this issue of the JCI, Adjali and colleagues report on their treatment of SCID mice lacking the 70-kDa protein tyrosine kinase, ZAP-70, with direct intrathymic injection of a ZAP-70-expressing T cell-specific lentiviral vector, which resulted in $T$ cell reconstitution (see the related article beginning on page 2287). Using lentiviral vectors and in situ gene transfer may represent a safer approach than using retroviral vectors for ex vivo gene transfer into HSCs, avoiding 3 factors potentially linked to leukemogenesis, namely HSC targets, ex vivo transduction and expansion, and standard Moloney leukemia virus-based retroviral vectors.
\end{abstract}

\section{SCID and its current standard of therapy}

SCID disorders are a family of genetically determined conditions characterized by a block in $\mathrm{T}$ cell development and function, variably associated with defects in other hematopoietic lineages. The genetic and phenotypic heterogeneity among these disorders is considerable. Affected children present with severe opportunistic infections due to a lack of functional mature $\mathrm{T}$ cells. The most serious forms of SCID are fatal in the first years of life (1). The only curative standard of therapy is an allogeneic bone

Conflict of interest: The authors have declared that no conflict of interest exists.

Citation for this article: J. Clin. Invest. 115:2064-2067 (2005). doi:10.1172/JCI26041. marrow transplant from an HLA-matched, related donor. If this is not possible, bone marrow transplantation from an HLAmatched, unrelated or a parental haploidentical donor is performed. The survival rate after HLA-matched transplantation is greater than $75 \%$ but falls markedly with the use of alternative donors, including haploidentical family donors and unrelated donors, and chronic disabling complications such as graft-versus-host disease are common. Some children reach full functional immunity following transplantation, but others fail to have their $\mathrm{B}$ cell function restored or can experience progressive loss of $\mathrm{T}$ cell immunity and even complete graft failure over time. These poor outcomes are particularly common when children are transplanted with non-HLA-matched donor cells, for instance from a parent or an unrelated donor $(2,3)$.

\section{Leukemia induction after successful oncoretroviral gene transfer in SCID-X1}

These unsatisfying outcomes, particularly with alternative donor transplantation, have stimulated intense interest in gene therapy approaches to SCID. The most common SCID subtype is SCID$\mathrm{X} 1$, an X-linked recessive disease characterized by a block in $\mathrm{T}$ and natural killer cell differentiation due to mutations in the common $\gamma$ chain of cytokine receptor complexes. Recently, complete immune reconstitution of young boys with SCID$\mathrm{X} 1$ by transduction of their $\mathrm{CD} 34^{+}$bone marrow cells with an oncoretroviral vector encoding the common $\gamma$ chain followed by reinfusion of transduced cells without conditioning therapy was reported (Figure 1A) $(4,5)$. These encouraging results represented the first unequivocal demonstration of the clinical efficacy of gene therapy. However, elation was short-lived, as 3 out of 11 enrolled children in this clinical trial have developed $\mathrm{T}$ cell leukemia linked to insertional mutagenesis, specifically, activation of the LIM domain only 2 (known as LMO2) transcription factor locus (6). Thus, at present the risks and potential benefits of gene therapy are being reconsidered. The 
A
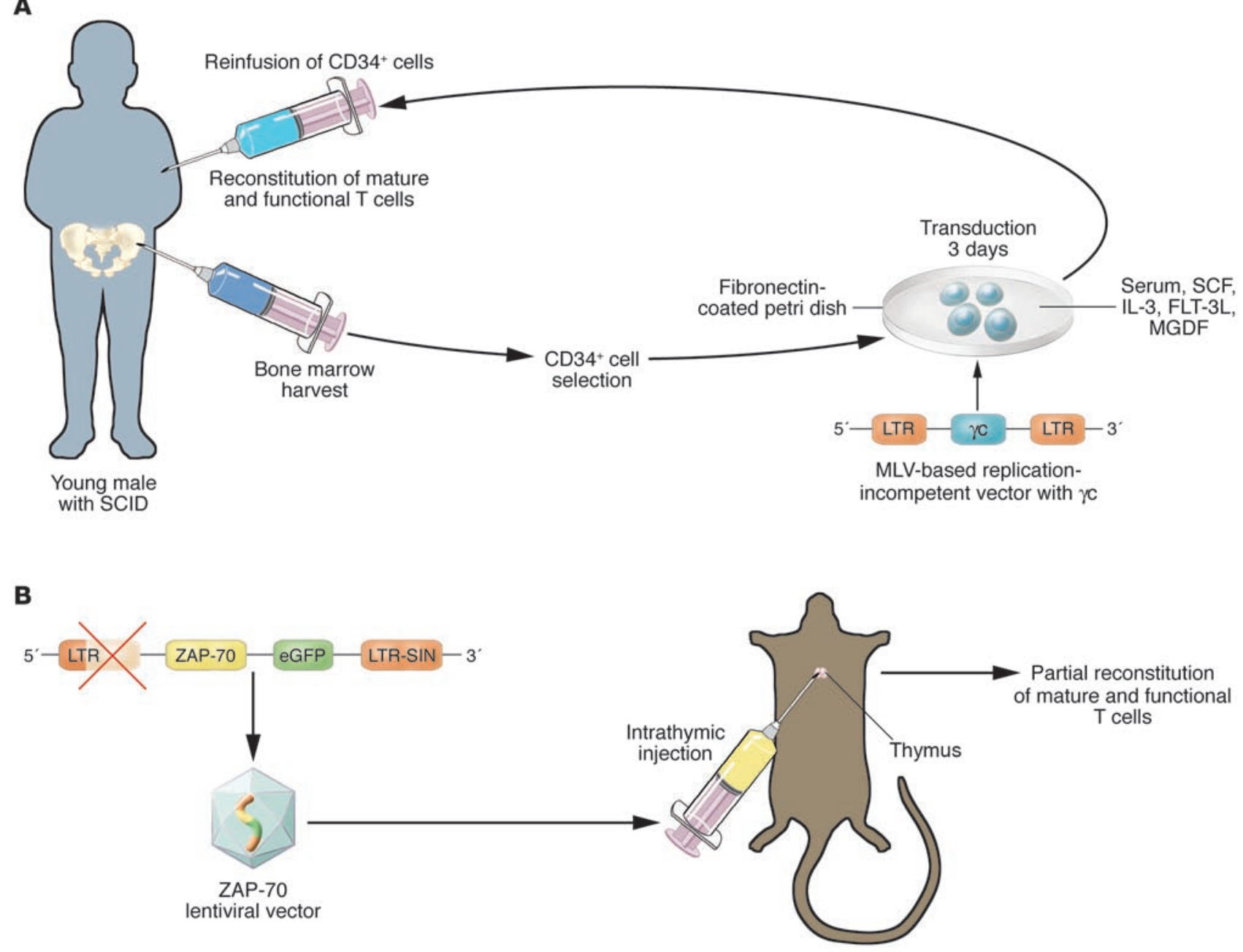

Figure 1

Gene therapy approaches for SCID. (A) Previously $(4,5)$, after informed parental consent, bone marrow was obtained from young boys with SCID-X1 who did not have an HLA-identical donor. Selection for the CD34+ cell population was performed, and these cells were stimulated to grow in media containing $4 \%$ fetal calf serum and supplemented with SCF, IL-3, Flt-3 ligand (FLT-3L), and megakaryocyte growth and differentiation factor (MGDF). The cells were transduced with a Moloney leukemia virus-based (MLV-based) replication-incompetent vector containing the common $\gamma$ chain $(\gamma c)$ for 3 days. The cells were reinfused into the patients without a preparative conditioning regimen. In the majority of the boys, mature and functional T cells were reconstituted. Unfortunately, 3 of 11 boys enrolled in this clinical trial developed a T cell leukemia related to the oncoretroviral vector used (6). (B) In this issue of the $\mathrm{JCl}$, Adjali and colleagues report (10) that they injected a T cell-specific lentiviral vector encoding human ZAP-70 and enhanced GFP (eGFP) directly into the thymi of 8 - to 12 -week old ZAP-70-- mice after thoracic surgery. Some of the mice partially reconstituted mature and functional T cells. LTR, long terminal repeat; LTR-SIN, self-inactivating LTR.

critical question is whether the high frequency of leukemia in this trial was related to specific features of these children or their target $\mathrm{CD}_{3} 4^{+}$cell populations, constitutive expression of this particular signaling transgene, or activity of vector elements such as enhancers or promoters as opposed to the possibility that these serious adverse events represent significant risks from the use of any integrating vector to transduce long-lived stem or progenitor cells.

The integration of replication-incompetent retroviruses was previously thought to be essentially random, and it was estimated that single copies of proviral genomes scattered throughout the genome were unlikely to result in the activation of protooncogenes. Recently, however, the insertion of oncoretroviral vectors has been shown to occur preferentially near transcription start sites of genes, potentially increasing the risk of altering the expression of adjacent endogenous genes, while lentiviral vectors were shown to integrate within genes even more preferentially, but over the entire length of transcription units instead of near transcriptional start sites, in a pattern possibly less likely to affect endogenous gene regulation (7-9). Insertional events related to both retroviral and lentiviral vectors are also enhanced in actively expressed genes. Current lentiviral vectors have deletions of their enhancer and promoter regions and instead use internal transgene-specific regulatory elements. Thus these vectors might be less likely to activate adjoining genomic transcription units than the strong constitutive regulatory elements present in the long terminal repeats of all currently utilized standard retroviral vectors (8). The lack of evidence of insertional leukemogenesis due to HIV integration in 
AIDS patients suggests that the risk may indeed be much lower for lentiviruses than for murine leukemia virus-derived retroviral vectors, but wild-type HIV can only infect mature $\mathrm{T}$ cells, not hematopoietic stem cells. Thus, the comparison may not be fully relevant.

\section{Alternative gene delivery approaches}

In this issue of the JCI, Adjali and colleagues (10) took a different approach to gene therapy for SCID. In this case, the authors targeted $T$ cell precursors instead of HSCs by direct intrathymic injection of a ZAP-70-expressing T cell-specific lentiviral vector into $Z A P-70^{-1-}$ SCID mice (Figure 1B). ZAP-70 deficiency is a rare autosomalrecessive inherited subtype of SCID, in which $\mathrm{T}$ cell development is blocked at the stage of double-positive cells, resulting in an absence of peripheral $\mathrm{CD}^{+} \mathrm{T}$ cells and the presence of circulating $\mathrm{CD}^{+} \mathrm{T}$ cells that are unresponsive to TCR-mediated stimuli in vitro (11). The differentation block in this disease is due to the inability of double-positive cells to propagate TCR signals in the absence of ZAP-70 kinase activity despite tyrosine phosphorylation of TCR-associated ZAP-70 molecules (12). The protein tyrosine kinase ZAP-70 is normally expressed in thymocytes, mature $\mathrm{T}$ cells, and natural killer cells (13).

Using the $T$ cell-specific lentiviral vector injected directly into the thymus of ZAP$70^{-1-}$ mice, Adjali and colleagues demonstrated partial reconstitution of polyclonal and functional $\mathrm{T}$ cells in some mice (10). They chose this approach to avoid transduction of HSCs and precursor cells from other hematopoietic lineages that do not express ZAP-70 and thus do not require correction in this disorder; this potentially reduces the risk of insertional mutagenesis by exposing many fewer precursor cells to insertional events. HSCs may be particularly susceptible to insertional mutagenesis due to their prolonged and increased replicative capacity. Many genes controlling self-renewal and proliferation are expressed in HSCs before being shut off during differentiation, and increased expression of genes has been shown to predispose loci to insertions of retroviral and lentiviral vectors $(7,9)$. Insertions at these sites could therefore constitutively activate genes that are normally shut off during hematopoietic development, and thus contribute to leukemogenesis. By the targeting of more mature cells via intrathymic injection, this risk might be decreased.
The in situ approach also avoids ex vivo culture of target cells, which is desirable for a number of reasons. Ex vivo culture of HSCs, progenitors, and even mature $\mathrm{T}$ cells has been found to result in impaired homing capabilities and functional deficits (14-16). Furthermore, ex vivo culture potentially exposes the cells to infectious agents, serum, and other risks related to complex and prolonged manipulations and removes them from their natural microenvironment. Thus, in situ delivery of a gene transfer vector to $T$ cell precursors without any requirement for ex vivo culture is an inherently attractive approach.

Although the lentiviral vector used in this study (10) was designed to be T cell-specific, it also transduced thymic epithelial cells (17). The interaction of thymocytes with thymic epithelial cells is crucial for the development of T cells. Thus the effects on thymic epithelial cell function by ZAP-70 transduction needs to be further evaluated. Changes in the vector design might be necessary to obtain increased $T$ cell specificity. Intrathymic vector injection is technically very challenging both in mice and humans but probably accounted for successful correction in less than a quarter of the mice treated by Adjali et al. (10). In human subjects suffering from SCID, the need for intrathymic injection will be a real hurdle, since in these patients the thymus is very small, usually less than $1 \mathrm{~g}$, and lacking in thymocytes (18). Thus, it might be difficult to establish this approach for clinical application, even if the success rate could be significantly improved via sophisticated imaging modalities and optimization in large animal models.

\section{Remaining questions and future directions}

The observation that long-term reconstitution of functional $\mathrm{T}$ cells is achievable using this approach in mice is encouraging. However, even though $\mathrm{T}$ cells can persist for many years, permanent reconstitution and continued production of naive $\mathrm{T}$ cells is thought to require correction of HSCs with self-renewing capacity. The durability of transduction of thymic early progenitor cells, which is likely responsible for the positive results in this study, is unclear (10). Longer follow-up studies are necessary for clarification. The ability of the $\mathrm{T}$ cell population to massively expand in number suggests the possibility that we could reconstitute the $\mathrm{T}$ cell compartment with a few gene-corrected cells, as seen after spontaneous mutation (19) or as demonstrated in this study (10). But is this the right strategy for reaching therapeutic levels of gene-corrected cells? Keeping in mind that expansion of a limited number of precursor cells and an abnormal proliferation advantage potentially contributed to the development of leukemia in the SCID-X1 trial, direct injection of vector into the thymus with subsequent marked expansion of cells now expressing ZAP-70 constitutively may have similar risks. The ZAP-70 transgene is a tyrosine kinase that may become oncogenic if its expression is not at physiologic levels in the appropriate hematopoietic lineages. Further modification of the lentiviral vector so that real lineage restriction and physiologic expression level of the transgene are achieved is probably desirable for improved safety. It will also be important to gain a better understanding of the biology of the thymic components in SCID patients in regard to the characteristics of the cells that are being transduced by the intrathymic injections, in particular if this approach is extended to other forms of SCID. Finally, longer term follow-up studies of many more mice as well as any SCID patients enrolled in trials using this approach will be crucial for understanding whether permanent correction is really possible via a non-HSC-based approach and for assessing leukemogenic risk.

Address correspondence to: Cynthia E. Dunbar, Molecular Hematopoiesis Section, Hematology Branch, National Heart, Lung, and Blood Institute, NIH/US Department of Health and Human Services, Building 10, Clinical Research Center, Room 4E-5132, 10 Center Drive, MSC1202, Bethesda, Maryland 20892, USA. Phone: (301) 496-1434; Fax: (301) 496-8396; E-mail: dunbarc@nhlbi.nih.gov.

1. Buckley, R.H., et al. 1997. Human severe combined immunodeficiency: genetic, phenotypic, and functional diversity in one hundred eight infants. J. Pediatr. 130:378-387.

2. Antoine, C., et al. 2003. Long-term survival and transplantation of haemopoietic stem cells for immunodeficiencies: report of the European experience 1968-99. Lancet. 361:553-560.

3. Patel, D.D., et al. 2000. Thymic function after hematopoietic stem-cell transplantation for the treatment of severe combined immunodeficiency. N. Engl. J. Med. 342:1325-1332.

4. Cavazzana-Calvo, M., et al. 2000. Gene therapy of human severe combined immunodeficiency (SCID)-X1 disease. Science. 288:669-672.

5. Hacein-Bey-Abina, S., et al. 2002. Sustained correction of X-linked severe combined immunodeficiency by ex vivo gene therapy. N. Engl. J. Med. 346:1185-1193.

6. Hacein-Bey-Abina, S., et al. 2003. LMO2-associated 
clonal $\mathrm{T}$ cell proliferation in two patients after gene therapy for SCID-X1. Science. 302:415-419.

7. Schroder, A.R., et al. 2002. HIV-1 integration in the human genome favors active genes and local hotspots. Cell. 110:521-529.

8. Wu, T., et al. 2000. Prolonged high-level detection of retrovirally marked hematopoietic cells in nonhuman primates after transduction of CD34+ progenitors using clinically feasible methods. Mol. Ther. 1:285-293.

9. Hematti, P., et al. 2004. Distinct genomic integration of MLV and SIV vectors in primate hematopoietic stem and progenitor cells. PLoS Biol. 2:e423.

10. Adjali, O., et al. 2005. In vivo correction of ZAP-70 immunodeficiency by intrathymic gene transfer. J. Clin. Invest. 115:2287-2295. doi:10.1172/JCI23966. 11. Elder, M.E. 1998. ZAP-70 and defects of T-cell receptor signaling. Semin. Hematol. 35:310-320.

12. Wiest, D.L., et al. 1997. A spontaneously arising mutation in the DLAARN motif of murine ZAP70 abrogates kinase activity and arrests thymocyte development. Immunity. 6:663-671.

13. Chan, A.C., et al. 1994. Differential expression of ZAP-70 and Syk protein tyrosine kinases, and the role of this family of protein tyrosine kinases in TCR signaling. J. Immunol. 152:4758-4766.

14. Habibian, H.K., et al. 1998. The fluctuating phenotype of the lymphohematopoietic stem cell with cell cycle transit. J. Exp. Med. 188:393-398.

15. Takatoku, M., et al. 2001. Avoidance of stimulation improves engraftment of cultured and retrovirally transduced hematopoietic cells in primates. J. Clin. Invest. 108:447-455. doi:10.1172/JCI200112593.

16. Sauce, D., et al. 2002. Retrovirus-mediated gene transfer in primary $\mathrm{T}$ lymphocytes impairs their anti-Epstein-Barr virus potential through both culture-dependent and selection process-dependent mechanisms. Blood. 99:1165-1173.

17. Marodon, G., and Klatzmann, D. 2004. In situ transduction of stromal cells and thymocytes upon intrathymic injection of lentiviral vectors. BMC Immunol. 5:18. http://www.biomedcentral. com/1471-2172/5/18.

18. Buckley, R.H. 2004. Molecular defects in human severe combined immunodeficiency and approaches to immune reconstitution. Annu. Rev. Immunol. 22:625-655.

19. Stephan, V., et al. 1996. Atypical X-linked severe combined immunodeficiency due to possible spontaneous reversion of the genetic defect in T cells. N. Engl. J. Med. 335:1563-1567.

\title{
Spring brings breezes, wheezes, and pollen oxidases
}

\author{
Darren R. Ritsick and J. David Lambeth
}

Department of Pathology and Laboratory Medicine, Emory University School of Medicine, Atlanta, Georgia, USA.

\begin{abstract}
While the release of pollen into the air is essential for the reproduction of plants, the accidental yet inevitable uptake of pollen into human airways can cause symptoms of seasonal allergies and asthma. The symptomatic response to pollen is caused by granulocytes that produce inflammation, which is due in part to oxidative stress through the action of NADPH oxidases. The recruitment of these inflammatory granulocytes was previously thought to depend entirely on the activation of an adaptive immune response. In this issue of the JCI, Boldogh et al. demonstrate that pollens contain endogenous NADPH oxidase activity, which functions to generate local "danger signals" in nearby airway epithelium (see the related article beginning on page 2169). These signals in turn trigger the early recruitment of granulocytes, even in the absence of the adaptive immune response. These findings suggest that inhibition of the pollen oxidase may provide a way to antagonize allergic inflammation at a very early step.
\end{abstract}

Spring... in the space of a few weeks, the drab winter grays give way to a riot of reds, pinks, yellows, and whites, as azaleas, dogwoods, and a host of other plants celebrate the arrival of longer, hotter days. But for many individuals, the bright banners of spring signal not a celebration, but a feeling of dread that augurs frequent trips to the pharmacy for the latest over-thecounter remedies. For amidst the color, another riot is taking place: in a reproductive frenzy, each member of the plant world is busily packaging its genome into protective capsules, which are ejected into the atmosphere or hitch rides on the bodies of insects. While a pollen count of 120

Conflict of interest: The authors have declared that no conflict of interest exists.

Citation for this article: J. Clin. Invest. 115:2067-2069 (2005). doi:10.1172/JCI26023. particles per cubic meter is considered high in many parts of the country, in the pollen belt of the southern US, counts of 6,000 are not unusual, and the accumulated particles confer a distinctly yellowish cast to the atmosphere.

While Mother Nature is merely assuring the reproductive success of her immobile plant kingdom by sowing the atmosphere with these tiny portable packages of DNA, the inadvertent consequence is that many of these particles do not find their intended targets but end up in the airways and mucous membranes of hapless humans. It is no wonder that the sounds of spring are punctuated by sneezes and coughs, and that the spring colors are seen through the blur of watery, itchy eyes. Indeed, as many as 35 million people in the US suffer from allergic reactions to pollens, ranging from annoying allergic rhinitis and allergic conjunctivitis to the more dangerous allergic asthma (1).

According to established paradigms, the pollen grains are interpreted by the host as invading foreign bodies, which results in a defensive immune reaction against the proteins and other biomolecules on the pollen's surface (2). This reaction involves inflammatory cells, including granulocytes such as eosinophils and mast cells, and can lead to chronic inflammation, airway hyperresponsiveness, bronchial constriction, increased fluid and mucus secretion, and in some cases, life-threatening sequelae (3). According to this conventional model, the inflammatory response includes the production of ROS via a membrane-associated superoxide-generating NADPH oxidase that is present in granulocytes (4, $5)$. This oxidase consists of the membranebound flavocytochrome $b_{558}$, of which gp91phox is the catalytic subunit, plus several regulatory subunits. When activated, the oxidase catalyzes the transfer of an electron from NADPH to molecular oxygen and forms superoxide $\left(\mathrm{O}_{2}{ }^{\circ}\right)$, with secondary production of hydrogen peroxide and other chemically reactive species (6, 7). Collectively, ROS can produce a wide range of molecular damage, which results in the oxidative modification of a variety of biomolecules as well as cellular damage and disruption of membrane integrity. In addition, ROS have more recently been implicated as intra- and intercellular signal molecules and therefore may participate 